\title{
Circulating endothelial progenitor cells are inversely correlated with the median oxygen tension in the tumor tissue of patients with cervical cancer
}

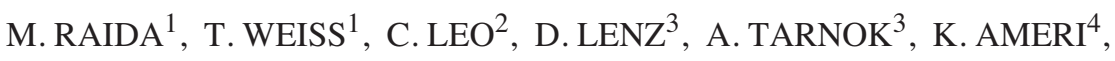 \\ A.L. HARRIS ${ }^{4}$, M. HÖCKEL ${ }^{2}$ and D. NIEDERWIESER ${ }^{1}$
}

\begin{abstract}
${ }^{1}$ Department of Hematology/Oncology, ${ }^{2}$ Department of Obstetrics and Gynecology, ${ }^{3}$ Interdisciplinary Center for Clinical Research Leipzig, University of Leipzig, D-04103 Leipzig, Germany; ${ }^{4}$ Cancer Research United Kingdom, Molecular Oncology Laboratory, Weatherall Institute of Molecular Medicine, John Radcliffe Hospital, Oxford OX3 9DS, UK
\end{abstract}

Received March 20, 2006; Accepted April 5, 2006

\begin{abstract}
Tumor hypoxia leads to adaptive responses in cancer cells, including an induction of vasculogenesis initiated by circulating endothelial progenitor cells (EPCs) and circulating endothelial cells (CECs). The aim of the present study was to correlate the number of EPCs and CECs with the oxygenation of cervical cancer. Blood concentrations of EPCs were detected by FACS analysis with antibodies for CD34 and vascular endothelial growth factor receptor 2 (VEGFR2). CECs were evaluated by double staining for 1,1'-dioctadecyl-3,3,3',3'tetramethylindocarbocyanine-labeled acetylated low density lipoprotein (Di-LDL) and lectin in a cell culture assay. Ten patients with cervical cancer were compared with ten healthy volunteers. Intratumoral oxygen tension was assessed polarographically with the computerized Eppendorf histography system. Analysis of CEC numbers revealed no difference between patients and controls. However, patients had lower concentrations of CD34-positive hematopoietic stem cells (HSCs) but a significantly higher fraction of EPCs related to the number of HSCs $(1.09 \%$ versus $0.53 \%)$. This fraction was significantly inversely correlated to the median oxygen tension ( $\mathrm{r}=-0.74, \mathrm{p}=0.015)$. Our study shows for the first time a significant inverse correlation between the fraction of EPCs and intratumoral oxygen tension. We conclude that the fraction of EPCs should be further evaluated as a useful and convenient marker in the prediction of tumor tissue oxygenation.
\end{abstract}

\section{Introduction}

Hypoxia is a reduction in the normal level of tissue oxygen tension which occurs during acute and chronic vascular disease,

Correspondence to: Dr M. Raida, Department of Hematology/ Oncology, University of Leipzig, D-04103 Leipzig, Germany

E-mail: martin.raida@medizin.uni-leipzig.de

Key words: endothelial progenitor, median oxygen tension, cervical cancer pulmonary disease and cancer. It induces a transcription programme that promotes an aggressive tumor phenotype (1). Hypoxia is associated with resistance to radiation therapy and chemotherapy, but is also associated with poor outcome regardless of treatment modality, indicating that it might be an important therapeutic target (2). Hypoxia represents the most potent activator of postnatal vascular growth and remodeling. Endothelial cells contribute to tumor angiogenesis, and can originate from sprouting or co-option of neighbouring preexisting vessels. The growth and metastasis of solid tumors correlate with their ability to induce the formation of new blood vessels. In addition to sprouting angiogenesis from neighboring resident endothelial cells, tumors also build vessels by recruitment and in situ differentiation of circulating endothelial progenitor cells (3-10). Bone marrow-derived endothelial precursors are necessary and sufficient for tumor angiogenesis (9), and EPCs differentiated ex vivo from multipotent adult progenitor cells, home to tumors (10). In cancer patients with no wounds or uterine cycling, neovasculogenesis is restricted to the growing tumor tissue and, therefore, recruitment of endothelial progenitors promises to be specific to the tumor.

The 'gold standard' for clinical assessment of the tumor oxygenation status remains at present the Eppendorf histography system, which involves the direct measurement of oxygen tension (oxygen partial pressure; $\mathrm{pO}_{2}$ ) using needle electrodes (11). However, the limitations of this technique are its invasiveness and lack of universal applicability because tumors undergoing investigation need to be accessed without substantial added risk for the patient or disproportionate effort on the part of the clinician. For these reasons, Eppendorf histography $\mathrm{pO}_{2}$ measurements are not feasible in many tumor entities and, thus, substitute endogenous or exogenous hypoxia markers would be of great interest in the clinical setting. In the present study, we investigated a possible correlation between the tissue oxygen tension of locally advanced cervical carcinomas and the number of EPCs and/or CECs.

\section{Materials and methods}

Patients and controls. Ten consecutive patients with cervical cancer presenting to the Department of Gynecology 
at Leipzig University between 2003 and 2004 (tumor size $\geq 2 \mathrm{~cm}$, determined clinically and with magnetic resonance imaging) formed the study group. Pretherapeutically, they underwent intratumoral oxygenation measurements with the Eppendorf histography system (Eppendorf, Hamburg, Germany), according to the standard procedure described previously (11), and $20 \mathrm{ml}$ of venous heparin blood was collected. The investigation of tumor oxygenation measurements was approved by the medical ethics committee of Leipzig University and all of the patients gave informed written consent. Ten healthy volunteers donated venous blood samples as a control group.

Tumor oxygenation measurements. Tumor oxygenation was measured with the Eppendorf histography system adhering to the standard procedure as developed and validated previously $(11,12) \cdot \mathrm{pO}_{2}$ readings were performed in the conscious patient along at least two linear tracks within the macroscopically vital tumor tissue. Within the tumor tissue, up to $35 \mathrm{pO}_{2}$ measurements were obtained in each tumor track starting at a tissue depth of $5 \mathrm{~mm}$. The measuring points were placed $0.7 \mathrm{~mm}$ apart, which resulted in an overall measurement track length of approximately $2.5 \mathrm{~cm}$. The median $\mathrm{pO}_{2}$ of all measured points was used to represent the oxygenation status of the respective tumor. Intravaginal temperature, blood pressure, heart rate, hemoglobin concentration, hematocrit and arterial oxyhemoglobin saturation were monitored concomitantly with the $\mathrm{pO}_{2}$ determinations.

Isolation, cultivation, and characterization of circulating endothelial cells. Mononuclear cells (MNC) were isolated by Ficoll density gradient centrifugation from $20 \mathrm{ml}$ of peripheral blood as described in the current literature (13). After isolation $2 \times 10^{6}$ cells were plated on gelatin-coated 4-well chamber slides (Nunc, Wiesbaden, Germany) in endothelial medium (Cell Lining, Berlin, Germany) containing 20\% fetal calf serum (Invitrogen, Karlsruhe, Germany), endothelial cell growth supplement (Cell Lining), and antibiotics (100 U penicillin $/ 100 \mu \mathrm{g} / \mathrm{ml}$ streptomycin; Invitrogen). After 4 days in culture, non-adherent cells were removed by a thorough rinsing with cell culture medium, and adherent cells were characterized by cytochemical methods.

Quantification of circulating endothelial cells with cell culture assay. To determine the amount of CECs among the adherent cells, the uptake of Di-LDL (Invitrogen) and the binding of FITC-labeled ulex europaeus agglutinin I (UEA-1) (Sigma, Munich, Germany) was analyzed (13). The number of CECs (UEA-1 and Di-LDL double-positive cells) was determined in an area of $200 \mathrm{~mm}^{2}$ by laser scanning cytometry (LSC) (CompuCyte, Cambridge, USA) $(14,15)$.

Quantification of circulating endothelial progenitor cells with flow cytometry analysis. Mononuclear cells (MNC) were isolated by density gradient centrifugation from $20 \mathrm{ml}$ of peripheral blood as described in the current literature (13). To quantify the content of circulating EPCs by FACS analysis, $6 \times 10^{6}$ cells were incubated for $15 \mathrm{~min}$ with allophyocyanin conjugated mouse anti-human CD34 (BD biotinylated antihuman VEGFR2 (R\&D Systems, Wiesbaden, Germany), cells

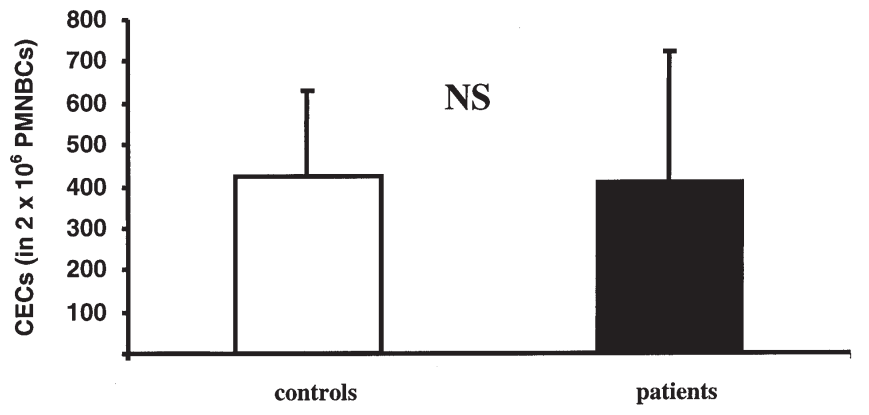

Figure 1. Comparison of circulating endothelial cells (CECs) in healthy controls $(n=10)$ compared to cervical cancer patients $(n=10 ; p=N S)$. Mean \pm standard deviation (SD) are given.

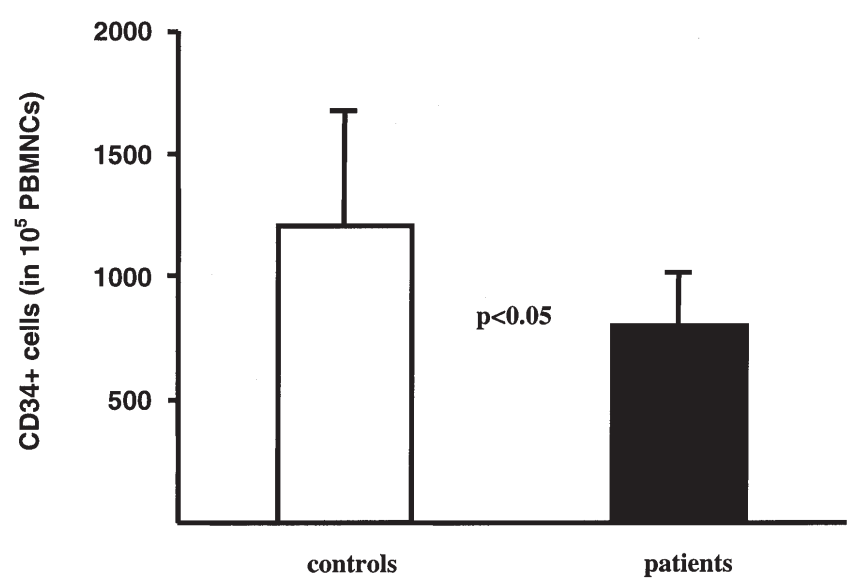

Figure 2. $\mathrm{CD}^{+} 4^{+}$hematopoietic stem cells (HSCs) in the peripheral blood of cervical cancer patients compared to healthy controls. Significantly lower concentrations of HSCs are detectable in the patients compared to the control individuals $(\mathrm{p}<0.05$, mean $\pm \mathrm{SD})$.

were washed with PBS for $5 \mathrm{~min}$ and incubated for $15 \mathrm{~min}$ with FITC-labeled streptavidin (DakoCytomation, Hamburg, Germany) in the dark. After incubation, the cells were washed with PBS and fixed in 2\% paraformaldehyde before analysis using a FACS Calibur (BD Biosciences). To quantify the amount of VEGFR2 ${ }^{+} \mathrm{CD} 34^{+}$double-positive cells, the mononuclear cell fraction was gated and analysed for the expression of CD34. Only the CD34-positive cells were finally investigated for the content of VEGFR2 ${ }^{+} / \mathrm{CD} 34^{+}$double-positive cells.

Statistical analysis. Statistical analysis was performed using the statistics package SPSS (version 11.5 for Windows; SPSS $\mathrm{GmbH}$, Munich, Germany). Correlations between two parameters were described by Spearman's rank correlation coefficient. T-test was used for comparison of categorized variables. A p-value $<0.05$ was considered to indicate statistical significance.

\section{Results}

Circulating endothelial cells (CECs) in healthy controls compared to cervical cancer patients. CECs were analysed with the cell culture assay in 10 healthy controls compared to cervical cancer patients. Adherent cells with an uptake of Di-LDL and binding of FITC-labeled UEA-1 were assessed as 

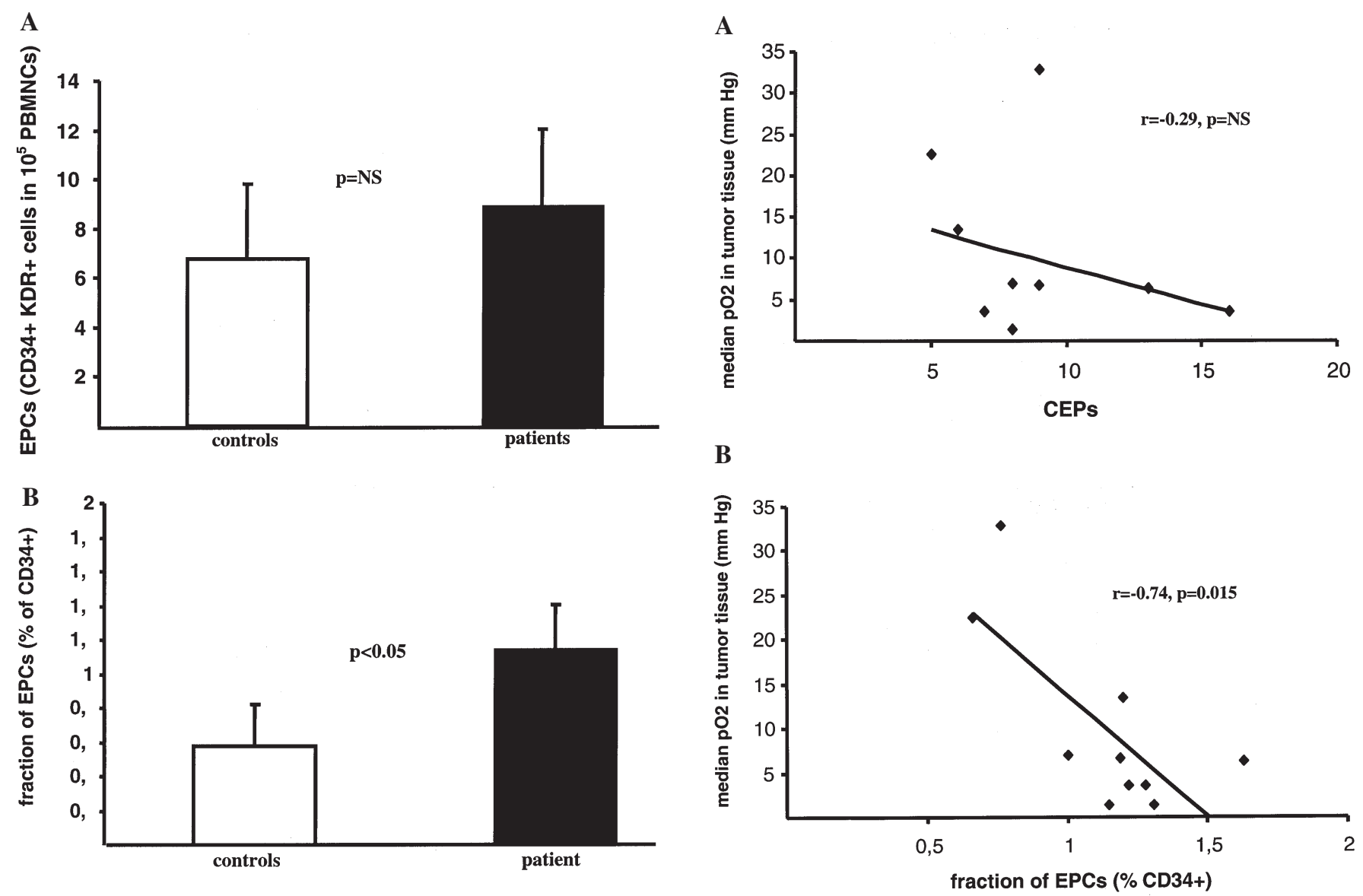

Figure 3. (A) FACS analysis of CD34 and VEGFR2 ${ }^{+}$circulating endothelial progenitor cells (EPCs) in cervical cancer patients compared to controls. The numbers of EPCs are similar in both groups. (B) Patients with cervical cancer showed a significantly higher fraction of EPCs related to number of HSCs ( $1.09 \%$ versus $0.53 \% ; \mathrm{p}<0.05$, mean $\pm \mathrm{SD})$.

CECs. Surprisingly, the amount of CECs detected with this assay in the peripheral blood of patients with cervical cancer showed no difference compared to healthy controls (Fig. 1).

Hematopoietic stem cells (HSCs) in the peripheral blood of cervical cancer patients compared to healthy controls. HSCs were detected in the peripheral blood with FACS analysis for $\mathrm{CD} 34^{+}$in cervical cancer patients and healthy controls. The concentration of HSCs was approximately $<50 \%$ in patients compared to control individuals $(\mathrm{p}<0.05)$ (Fig. 2). We then looked at the presence of EPCs by measuring $\mathrm{CD}_{3} 4^{+}$and VEGFR2 ${ }^{+}$cells in the peripheral blood. Similar numbers of EPCs were found in cervical cancer patients compared to controls (Fig. 3A). However, patients with cervical cancer showed a significantly higher fraction of EPCs related to the total number of HSCs $(1.09 \%$ versus $0.53 \%, \mathrm{p}<0.01)$ (Fig. 3B).

EPCs in the peripheral blood of patients with cervical cancer and correlation to intratumoral oxygen tension. The total number of EPCs in the peripheral blood of patients with cervical cancer revealed no significant correlation to the median intratumoral oxygen tension (Fig. 4A). When the fraction of EPCs was related to the total number of HSCs, a significantly inverse correlation to the median intratumoral oxygen tension (Fig. 4B) could be demonstrated.

Figure 4. (A) The number of EPCs in the peripheral blood of patients with cervical cancer reveals no significant correlation to the median intratumoral oxygen tension. (B) The fraction of EPCs related to the number of HSCs is significantly inversely correlated to the median intratumoral oxygen tension.

\section{Discussion}

The tumor oxygenation status is an independent prognostic indicator in cancer because it influences tumor progression and treatment outcome $(2,12)$. The measurement and interpretation of oxygenation data obtained with currently available methods is complicated by the heterogeneity of oxygen tension in the tumor tissue. Currently available techniques can be divided into direct invasive methods, direct non-invasive methods, and measurement of surrogate endogenous markers of tumor oxygenation status. Eppendorf $\mathrm{pO}_{2}$ histography is still considered as the 'gold standard' although it has several limitations. Given the importance of tumor hypoxia in antitumoral therapy and in predicting tumor progression, a technically simplistic method is needed to obtain a reliable profile of tumor oxygenation. To the best of our knowledge, this study demonstrates for the first time a significant inverse correlation between EPCs and intratumoral oxygenation in cervical cancer patients. However, it was not the total number of CD34/VEGFR2-positive circulating EPCs detected by FACS analysis but the fraction of EPCs related to the total number of HSCs that was significantly inversely correlated to the median intratumoral oxygen tension. Thus, the number of EPCs in the peripheral blood was similar in both groups due to a higher concentration of HSCs in healthy controls compared to patients with cervical cancer. Unexpectedly, the amount of lectin and Di-LDL positive 
CECs assessed with an adhesion cell culture assay showed no difference in patients with cervical cancer compared to healthy controls. Our data is in conflict with findings reported in breast cancer and lymphoma patients, that demonstrated a 5-fold increase of CEC numbers compared to healthy controls by means of flow cytometry (16). A more recent study showed that cancer patients with progressive disease had, on average, 3.6-fold more CECs than healthy subjects (17). There are several explanations for the discrepancy of our results in comparison to the literature. First of all, the small number of our study population was due to the technically demanding oxygen measurement procedures, making it difficult to provide a valid estimation of the average CEC number in untreated cervical cancer patients. Additionally, differences in the techniques applied for the quantification of CECs might also add to the conflicting results. We used a quantitative slide based assay with laser scanning cytometry while most of the other publications used a FACS based detection technique for the quantification of CECs. It is also important to notice that our untreated patient population was not characterized concerning the dynamic of tumor progression. In this respect, Beerepoot and co-workers observed that patients with stable disease had CEC numbers equal to those circulating in healthy subjects (17). Another interesting aspect is the fact that GM-CSF treatment has been shown to induce a CEC increase, probably by mobilization from the bone marrow (18). In our study, untreated patients with cervical cancer had $50 \%$ lower numbers of HSCs compared to healthy control individuals, indicating a suppression of bone marrow function. Only the fraction of EPCs related to the total number of HSCs was significantly inversely correlated to the median intratumoral oxygen tension. We would, therefore, suggest to normalize EPC and CEC numbers to the peripheral HSC count.

There is strong evidence in the literature that endothelial progenitor cell recruitment is closely related to tissue oxygenation. Recently, it has been shown that the expression of the chemokine stromal cell-derived factor-1 (SDF-1) which mediates homing of stem cells to bone marrow is regulated by the transcription factor hypoxia-inducible factor-1 (HIF-1) in endothelial cells, resulting in selective in vivo expression of SDF-1 in ischemic tissue in direct proportion to reduced oxygen tension (19). In bone marrow, the normal physiologic oxygen tension is hypoxic and SDF-1 is constitutively expressed, which provides an optimal niche for stem and progenitor recruitment. When a tumor is growing, soluble factors, such as SDF-1 and vascular endothelial growth factor (VEGF), are released by hypoxic tumor tissue areas and stimulate mobilization of progenitor cells from the bone marrow. These cells are then recruited to hypoxic tumor tissue by high local levels of SDF-1, providing a permissive niche (19). During conditions of ongoing local tumor tissue hypoxia, progenitor cells proliferate and assemble into vascular structures, contributing to remodeling of the nascent vascular network.

For many years, tumor hypoxia has been recognized as a potential therapeutic problem because of its adverse impact on the effectiveness of radiation therapy. However, hypoxia has recently been identified as an important factor that influences tumor proliferation and malignant progression (20). Although some of the effects of hypoxia negatively impact tumor cell growth (1), they may, antithetically, lead to hypoxia-driven responses that enhance malignant progression and aggressiveness, ultimately resulting in increased resistance to therapy and a poor long-term prognosis. The detection of intratumoral oxygen tension is technically demanding and not applicable for routine use in most of the solid tumors. Although our study was performed in a small study population of cervical cancer patients, it demonstrates for the first time a significant inverse correlation between the fraction of EPCs and intratumoral oxygen tension. We conclude, therefore, that the fraction of EPCs should be further explored concerning its potential value as a convenient surrogate marker of tumor oxygenation, which is important in the prediction of responsiveness to antitumoral strategies that rely on a certain tissue oxygenation as well as for the response monitoring of anti-angiogenic therapies.

\section{Acknowledgments}

We thank Dr Volker Adams and Christine Günther for their technical expertise and help in performing the endothelial cell assays.

\section{References}

1. Harris AL: Hypoxia - a key regulatory factor in tumour growth. Nat Rev Cancer 2: 38-47, 2002.

2. Vaupel P, Kelleher DK and Hockel M: Oxygen status of malignant tumors: pathogenesis of hypoxia and significance for tumor therapy. Semin Oncol 28: 29-35, 2001.

3. Hanahan D and Folkman J: Patterns and emerging mechanisms of the angiogenic switch during tumorigenesis. Cell 86: 353-364, 1996.

4. Asahara T, Murohara T, Sullivan A, Silver M, van der Zee R, Li T, Witzenbichler B, Schatteman G and Isner JM: Isolation of putative progenitor endothelial cells for angiogenesis. Science 275: 964-967, 1997

5. Asahara T, Masuda H, Takahashi T, Kalka C, Pastore C, Silver M, Kearne M, Magner M and Isner JM: Bone marrow origin of endothelial progenitor cells responsible for postnatal vasculogenesis in physiological and pathological neovascularization. Circ Res 6: 221-228, 1999.

6. Shi Q, Rafii S, Wu MH, Wijelath ES, Yu C, Ishida A, Fujita Y, Kothari S, Mohle R, Sauvage LR, Moore MA, Storb RF and Hammond WP: Evidence for circulating bone marrow-derived endothelial cells. Blood 92: 362-367, 1998.

7. Peichev M, Naiyer AJ, Pereira D, Zhu Z, Lane WJ, Williams M, Oz MC, Hicklin DJ, Witte L, Moore MA and Rafii S: Expression of VEGFR-2 and AC133 by circulating human CD34(+) cells identifies a population of functional endothelial precursors. Blood 95: 952-958, 2000.

8. Gehling UM, Ergun S, Schumacher U, Wagener C, Pantel K, Otte M, Schuch G, Schafhausen P, Mende T, Kilic N, Kluge K, Schafer B, Hossfeld DK and Fiedler W: In vitro differentiation of endothelial cells from AC133-positive progenitor cells. Blood 95: 3106-3112, 2000.

9. Lyden D, Hattori K, Dias S, Costa C, Blaikie P, Butros L, Chadburn A, Heissig B, Marks W, Witte L, Wu Y, Hicklin D, Zhu Z, Hackett NR, Crystal RG, Moore MA, Hajjar KA, Manova K, Benezra R and Rafii S: Impaired recruitment of bonemarrow-derived endothelial and hematopoietic precursor cells blocks tumor angiogenesis and growth. Nat Med 7: 1194-1201, 2001.

10. Reyes M, Dudek A, Jahagirdar B, Koodie L, Marker PH and Verfaillie CM: Origin of endothelial progenitors in human postnatal bone marrow. J Clin Invest 109: 337-346, 2002.

11. Höckel M, Schlenger K, Knoop C and Vaupel P: Oxygenation of carcinomas of the uterine cervix: evaluation by computerized $\mathrm{O}_{2}$ tension measurements. Cancer Res 51: 6098-6102, 1991.

12. Höckel M, Schlenger K, Aral B, Mitze M, Schäffer U and Vaupel P: Association between tumor hypoxia and malignant progression in advanced cancer of the uterine cervix. Cancer Res 56: 4509-4515, 1996. 
13. Vasa M, Fichtlscherer S, Aicher A, Adler K, Urbich C, Martin H, Zeiher AM and Dimmeler S: Number and migratory activity of circulating endothelial progenitor cells inversely correlate with risk factors for coronary artery disease. Circ Res 89: 1-7, 2001.

14. Tarnok A and Gerstner AO: Clinical applications of laser scanning cytometry. Cytometry 50: 133-143, 2002.

15. Lenz D, Lenk K, Adams V, Boldt A, Hambrecht R and Tarnok A: Detection of endothelial progenitor cells by flow and laser scanning cytometry. In: Manipulation and Analysis of Biomolecules, Cells, and Tissues. Nicolau DV, Enderlein J, Leif RC and Farkas DL (eds). Proc SPIE 4962: 384-394, 2003.

16. Mancuso P, Burlini A, Pruneri G, Goldhirsch A, Martinelli G and Bertolini F: Resting and activated endothelial cells are increased in the peripheral blood of cancer patients. Blood 97: 3658-3661, 2001.
17. Beerepoot LV, Mehra N, Vermaat JS, Zonnenberg BA, Gebbink MF and Voest EE: Increased levels of viable circulating endothelial cells are an indicator of progressive disease in cancer patients. Ann Oncol 15: 139-145, 2004.

18. Takahashi T, Kalka C, Masuda H, Chen D, Silver M, Kearney M, Magner M, Isner JM and Asahara T: Ischemia and cytokineinduced mobilization of bone marrow-derived endothelial progenitor cells for neovascularization. Nat Med 5: 434-438, 1999.

19. Ceradini DJ, Kulkarni AR, Callaghan MJ, Tepper OM, Bastidas N, Kleinman ME, Capla JM, Galiano RD, Levine JP and Gurtner GC: Progenitor cell trafficking is regulated by hypoxic gradients through HIF-1 induction of SDF-1. Nat Med 10: 858-864, 2004.

20. Bacon AL and Harris AL: Hypoxia-inducible factors and hypoxic cell death in tumour physiology. Ann Med 36: 530-539, 2004. 